\title{
Adoption and Use of Emerging Cultural Technologies in China's Museums
}

\author{
Eugene $\mathrm{Ch}^{\prime} \mathrm{ng}^{1}$, Shengdan $\mathrm{Cai}^{1}$, Fui-Theng Leow ${ }^{2}$ and Tong Evelyn Zhang ${ }^{1}$
}

eugene.chng@nottingham.edu.cn; shengdan.cai@nottingham.edu.cn; fui-theng.leow@nottingham.edu.cn; evelyn.zhang@nottingham.edu.cn

${ }^{1}$ NVIDIA Joint-Lab on Mixed Reality, University of Nottingham Ningbo China

${ }^{2}$ Faculty of Business, University of Nottingham Ningbo China

\begin{abstract}
Our article maps the well-being of the adoption and use of digital technologies in museums in China. Digital technologies used here are termed 'cultural technology' in top-level national policies for the creative cultural industry, with culture and heritage at the core of all such activities. Our investigation aims to understand the present state of adoption and use of cultural technologies with the goal of identifying limitations so as to provide a roadmap for the informed design and development of museum-based digital exhibits that are appropriate for visitors. Whilst our study is focused on China's museums, our evaluation model and lessons learned can be used as comparative studies for museums globally. This highlights the novelty of our article, for the scale of which we carried out our evaluation has never been conducted before. We travelled 22 sites over 15 cities and collected over 800 samples of data. We evaluated 36 digital systems used by over 800 visitors and observed how users interacted and engaged with the systems with a record of 21 variables related to the length of interaction, engagement, quality of contents and types of systems, age groups, sexes, and the number of participants and whether they were individuals or in groups. Our investigation revealed important findings in both digital systems and visitor engagement.
\end{abstract}

Keywords: digital museum, museum computing, digital heritage, smart museum, cultural technology, virtual reality, augmented reality, China

\section{Introduction}

Emerging digital technologies is a key factor for the sustainable preservation and communication of cultural heritage for mass audiences and is core to generating value in activities that contribute to the creative economy, at least in China. China's top-down policies such as the Cultural Technology Innovation Plan [1] aims to 'fully facilitate the integration of scientific technology into the cultural sector, and the '13th Five Year Plan' (2016-2020) proposed to 'build the cultural industries as a national pillar industry' [2]. Our study will serve as a snapshot of an important intersection in time for the years of expected development to come.

The Chinese government has, in the last decade exerted influence and emphasis on the development of the cultural industry. This has progressed from the setting of goals to actively "develop the cultural industry as a pillar industry of the national economy" in the previous '12 th Five Year Plan', to a projection stating that the "cultural industry will become a pillar industry of national economy at the end of the '13 th Five Year Plan"' [2].

In view of such a plan, we focused our evaluation on cultural technologies in an important sector within the creative cultural industry. This is the museum sector where 
major cultural artefacts and histories are curated and contextualised. In our observations, we noted that an increasing number of emerging digital technologies are being adopted in museums which we hypothesised to be object-centric, and not designed with users in mind. That is to say, the design of digital exhibits is top-down and focused on objects rather more than the needs of visitors. The lack of user studies is confirmed with our informal interviews and discussions working with museum delegates. As such, it has become a priority to evaluate the adoption and use of digital technologies within China's cultural institutions as a means to understand their digital well-being, and the distance from the success of fulfilling the ambitious plans which China has set forth for its key cultural sector. In the West, most evaluations have been at the device level, conducted within a single museum. These were studies aiming at depth of understanding of contents and how users have engaged with them via user interfaces. We believe that the breadth of evaluation of adoption and use is needed to understand and benchmark the overall health of digital technology adoption and use.

Understanding the breadth of adoption and the use of digital technologies in museums will reveal challenges and opportunities in relation to China's strategic national plans and the cultural creative industry, and China's standing more globally.

\section{Background}

The use of technology in museums dates back to the late 1960s, during which the majority of systems were used for documentation and management of museum collections. Ross Parry's [3] review of museum computing history shows the advent of communication role of museums in light of media theories [4,5] at the beginning of the 1970s. In the decades to follow, the rise of information and communications technologies saw a paradigm shift in the museum agenda, the nature of museums transitioned from having "object centredness" to visitor experience [6,7]. This shift is a catalyst to a discourse on the relationship between newly developed technologies, museum interpretation and visitor experience.

The onset of technological innovations (mobile and handhelds, multitouch screens, interactive 3D, Augmented Reality and Virtual Reality) and the increasingly creative use of such technologies have generated tremendous amounts of discussions. In the academia, applications of pioneering works continue to bring to light the transformative nature of such technologies for art and cultural heritage.

Most published research works were project-oriented with an aim to achieve best practices for museums, i.e., evaluation of touch screens-based information kiosks in art, history and archaeology museums [8], [9]. There are research related to the qualitative evaluation of multitouch screens [10] and gaze analysis for stereo interactive 3D multitouch screens [11], digital augmentation device [12], and handheld guide device in a historic house [13]. These researches have leaned towards the advantages of digital installations, providing contextualised information, and enhanced interactions and engagement within exhibitions [9]. However, we should always be cautious with regards to the use of new technologies for cultural heritage [14].

Published studies [9], [13], [12] for learning about visitor behaviours and for the evaluation of digital systems were mainly conducted in the West (see [13], [15], [16]). There have been very few studies for museums in the East. As far as we know, there have not been any formal studies of museums in China except for a single case study of an online Chinese digital science and technology museum [17]. We surveyed Mandarin- 
language literatures and discovered that the majority of articles were either a discussion of the concept of what digital museums are [18][19], or have been proposed recommendations of how museums should deploy digital technologies for exhibitions $[20,21]$. These works mainly emphasised the advantages of digital exhibits and their affordances of interactivity and multi-sensory experience. Ironically, these deeper discussions of the advantages of digital technologies have not been evaluated with visitors in mind, and from the perspectives of users, with one exception [22]. Liu's study surveyed the public use and understanding of digital exhibits within museums and revealed that only $17.14 \%$ respondents felt satisfied with the current installation of multimedia systems. Participants that were opposed to the current form of exhibits often have a preference for, and expectations of "interactive games", "Virtual Reality", "Scenario Reconstructions" and "Interactive Exhibit Design" which, according to our evaluation reported in this article have not been appropriately nor adequately adopted.

\section{Methodology}

Here, we designed a model for conducting a broad, cross-site evaluation of museums.

\subsection{Sites and Museums}

Our study examines national and local museums across China (Figure 1). We conducted observations during the weekends so that our sample yields are greater.

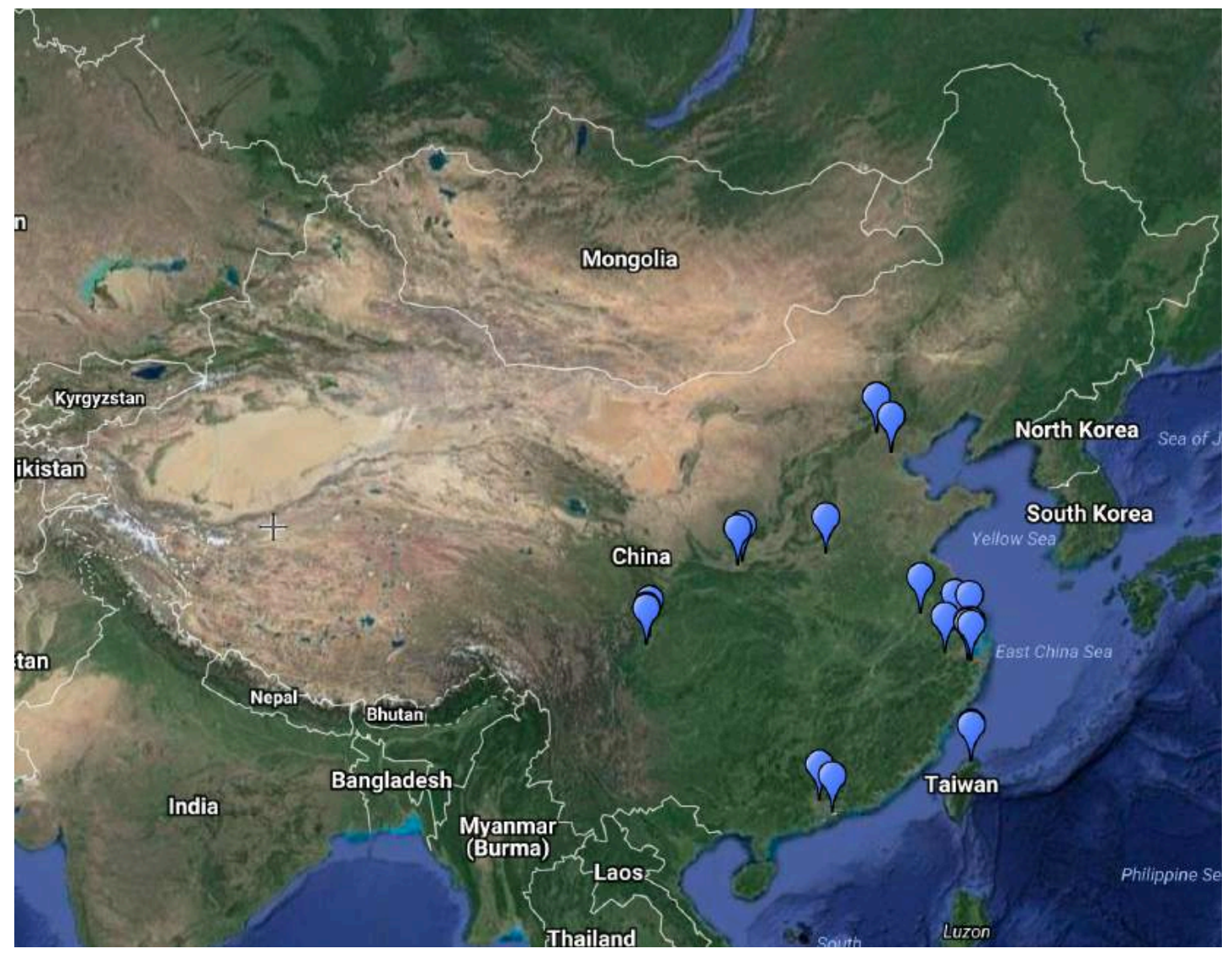

Figure 1. The map of our evaluation which covers 22 museum and sites across 15 different cities 
Table 1 is the corresponding sample size collected from each museum. Some museums do not have digital systems but are included as part of our study for the sake of recording their non-adoption of technologies. Others, such as the Palace Museum and the Shaanxi History Museum yielded zero samples as the digital systems were either under construction or had signs saying that they were still in development. Nevertheless, these do count as samples within our study as they can reveal insights when our datasets have been analysed.

\begin{tabular}{|c|c|c|c|}
\hline Date (2017) & Location & Museum & Samples \\
\hline $\begin{array}{l}\text { Mon } 23 \text { Jan }- \\
\text { Wed 25 Jan }\end{array}$ & Taipei & $\begin{array}{l}\text { National Palace Museum } \\
\text { National Museum of History }\end{array}$ & $\begin{array}{l}71 \\
22\end{array}$ \\
\hline Fri 3 Feb & Ningbo & Baoguo Temple & 0 \\
\hline $\begin{array}{l}\text { Wed } 15 \text { Feb }- \\
\text { Fri } 17 \text { Feb }\end{array}$ & Shanghai & Shanghai Museum & 61 \\
\hline $\begin{array}{l}\text { Fri } 10 \text { Mar - Sun } \\
12 \mathrm{Mar}\end{array}$ & Beijing & $\begin{array}{l}\text { National Museum of China } \\
\text { The Palace Museum }\end{array}$ & $\begin{array}{c}41 \\
0\end{array}$ \\
\hline $\begin{array}{l}\text { Fri } 31 \text { Mar - Tue } \\
4 \text { Apr }\end{array}$ & $\begin{array}{l}\text { Chengdu } \\
\text { Guanghan }\end{array}$ & $\begin{array}{l}\text { Jinsha Site Museum } \\
\text { WuHou Shrine Museum } \\
\text { Sanxingdui Museum }\end{array}$ & $\begin{array}{l}62 \\
30 \\
28\end{array}$ \\
\hline $\begin{array}{l}\text { Fri } 31 \text { Mar }- \\
\text { Mon } 3 \text { Apr }\end{array}$ & $\begin{array}{l}\text { Nanjing } \\
\text { Suzhou }\end{array}$ & $\begin{array}{l}\text { Nanjing Museum } \\
\text { Suzhou Museum }\end{array}$ & $\begin{array}{l}86 \\
84\end{array}$ \\
\hline Sat 8 April & Yuyao & $\begin{array}{l}\text { Hemudu Site Museum } \\
\text { Tianluoshan Site Museum }\end{array}$ & $\begin{array}{l}0 \\
0\end{array}$ \\
\hline Mon 1 May & Ningbo & Ningbo Museum & 62 \\
\hline $\begin{array}{l}\text { Fri } 5 \text { May - Sun } \\
7 \text { May }\end{array}$ & Tianjin & $\begin{array}{l}\text { Tianjin Museum } \\
\text { Tianjin Natural History Museum }\end{array}$ & $\begin{array}{l}23 \\
92 \\
\end{array}$ \\
\hline $\begin{array}{l}\text { Fri } 5 \text { May - Mon } \\
8 \text { May }\end{array}$ & $\begin{array}{l}\text { Shenzhen } \\
\text { Guangzhou }\end{array}$ & $\begin{array}{l}\text { Shenzhen Museum, } \\
\text { Guangdong Museum }\end{array}$ & $\begin{array}{l}41 \\
30 \\
\end{array}$ \\
\hline $\begin{array}{l}\text { Fri } 12 \text { May - } \\
\text { Mon } 15 \text { May }\end{array}$ & $\begin{array}{l}\text { Xi'an } \\
\text { Zhengzhou }\end{array}$ & $\begin{array}{l}\text { Emperor Qinshihuang's Mausoleum Museum, } \\
\text { Shaanxi History Museum, } \\
\text { Henan Museum }\end{array}$ & $\begin{array}{l}0 \\
0 \\
0\end{array}$ \\
\hline $\begin{array}{l}\text { Sat } 28 \text { May - } \\
\text { Sun } 30 \text { May }\end{array}$ & Hangzhou & Zhejiang Museum (Gushan and Wulin) & 74 \\
\hline Total & 15 & 22 & 807 \\
\hline
\end{tabular}

Table 1: A list of sites and museums and the sample sizes collected

\subsection{Identifying Digital Systems for Observation}

Our criteria were that systems should be digital at very the least, with some form of interactivity in the user interfaces. Projection systems with narratives were part of our study. Videos such as documentaries were excluded. In our categorisation, we looked for the adoption of popular technologies listed below: 
- Augmented Reality (AR) - any devices which augment virtual objects onto the real world using QR code, images, or spaces (e.g., HoloLens)

- Virtual Reality (VR) - any displays which completely immerses a user into a virtual world (e.g., headsets, CAVE), this includes 360 videos

- Projection Displays - displays which provide a narrative and are not only a video

- Interactive 2D - this includes 2D interactive systems and 1990s era multimedia systems or touch screens

- Multitouch2D - this includes multiuser, multitouch displays which supports at least two users within a single session of use.

- Interactive 3D - 3D interactive environments either with an interactive device (i.e., Mouse) or touch screens and gestures

- Mobile Exhibit - a mobile device (i.e., mobile phones, iPad, etc.)

- Miscellaneous - any unexpected interactive devices

The last two elements of our categories 'Mobile Exhibits' and 'Miscellaneous' were not found within the sites and museums we have evaluated.

\subsection{Quality of Digital Exhibits}

We designed a strategy for observing digital exhibits and their use based on both quantitative observations via the collection of data and qualitative analysis.

We browsed through each exhibit and recorded the types, nature of contents, length of time needed to completely browse through contents (accessing links, reading texts, watching videos, etc.) and the quality of exhibits in term of their overall designs, user interfaces, navigation approaches and contents. Whilst the variable 'Quality of Exhibit' is a subjective evaluation, our team members have expertise in the design, development and use of many interactive systems. The quality of a system is determined by its overall look and feel, the effectiveness of its user interfaces in carrying out the intended tasks, the efficiency of navigating contents, the relevance of the contents to a particular exhibition, its retention power and the general experience accessing such systems which may include excitable contents.

Biases were minimised via debriefing sessions and where needed, video captures of the systems were reviewed. The length of exhibits is important for judging how engaging the digital systems fared. For example, a system that was used for 1 minute that was originally designed for 15 minutes is probably not engaging, and is not used to the fullest.

We noted that the popularity of an exhibit does depend on the popularity of the museum itself, and that the type of contents is as important as the popularity of the device. However, the fact that an exhibit is in demand does have important implications to the successful adoption of a piece of digital technology and its content design. This is an indirect means of observation for determining how popular an exhibit is.

\subsection{Evaluating the Use of Digital Exhibits}

We kept our observations covert in order to not affect nor disturb the users. Apple iPads with a split screen (see Figure 2) were used for recording data. 


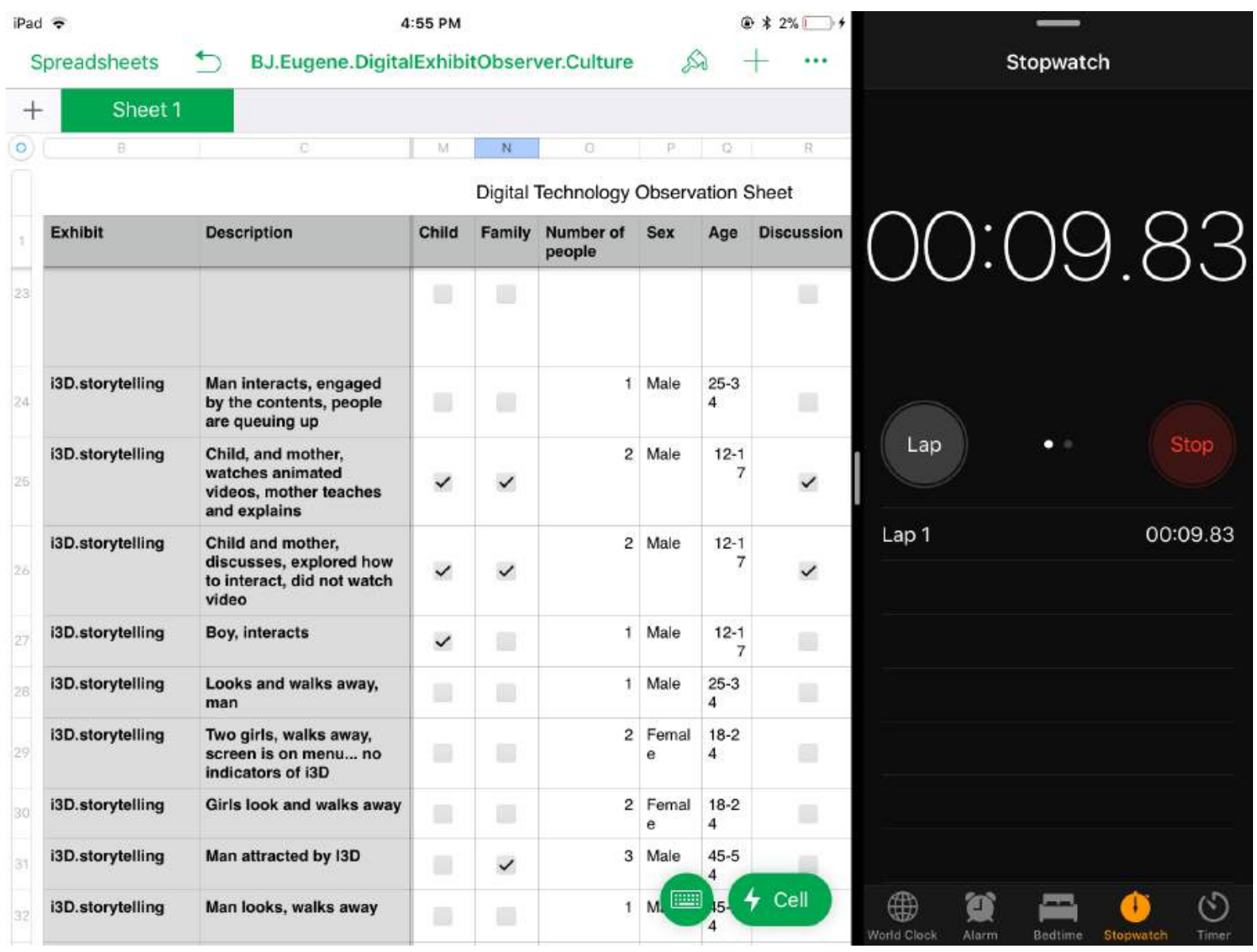

Figure 2. Observations and recording using a split screen iPad

For each digital system, we recorded 30 samples of use or up to 2 hours of observations whichever comes first. A system yielding 30 samples in 30 minutes may be judged as a popular system. For some museums with more digital systems, we also added a rule to record only 20 samples or up to 1 hour of observations so that we cover more types of exhibits. Our total sample size for each museum counts towards our aim of evaluating the breadth of technology adoption across an entire country.

\subsubsection{Digital Exhibits}

This section describes the variables associated with a single digital exhibit.

Exhibit ID - coding for identity, e.g., i2D.Beijing.Storytelling, i3D.Chengdu.HeroModels, and etc.

Location of Exhibit - the name of the museum and the city.

Length of Exhibit - the length of time it took to browse through all contents, including the length of a video, reading through texts, accessing links, interaction and etc.

Relevance to Contents - the system's use of contents and how it differs from the 
subject. If a system introduces superfluous, unnecessary contents or interfaces that do not contribute to the learning of the contents, the system is judged as not relevant (1), a score of 5 has highly relevant contents, but the contents may be purely informational.

Quality of Exhibit - the quality of the exhibit in terms of its overall design, user interface, system navigation, and 2D/3D contents.

Info - used for recording our thoughts and comments on the digital system.

\subsubsection{User Demographics}

In cases where visitor groups were observed, the sex of the dominant person is recorded. Age is difficult to judge without deliberately asking the user which we strictly refrained from. We therefore binned the age range: $<=12,12-17,18-24,25-34,35-44$, $45-54,55-64,65-74,>=75$. As our users are predominantly Chinese visitors, the age of the dominant person has not been difficult to estimate.

\subsubsection{Social Interaction Around Digital Exhibits}

The category of data records the social aspects of the use of a digital exhibit.

Family - is ticked if the group appears to be a family, which are usually parents and children and occasionally relatives as determined by conversations.

Discussion - a binary value which records any discussions taking place.

Teaching - if teaching and learning took place between the members of the group.

Guided Tour - is ticked if there is a tour guide leading a group, usually a large group of more than 5 persons.

Photo Taking - is ticked if the users took photos of the exhibit.

\subsubsection{Engagement and Interaction}

This section describes user interactions and engagement with an exhibit.

Description - a record of occurrences during user interactions with as many details as possible for verifying our observations during our debriefing sessions. This includes discussions, user interactions and observed group behaviour.

Is Crowded - the space around the exhibit is crowded.

Queued - for describing situations in which a person intends to use the exhibit queued earlier and subsequently used the exhibit.

Attracts Queue - is observed when a person uses the exhibit and attracts other users to watch or wait. 
Time at Exhibit - records the length of time a user spent on the exhibit.

Engagement/Interface - is a subjective observation of a user's or groups' engagement with the content/interface. The description variable provided the details but this variable ranks between 1 being weak and 5 as strong engagement. The TimeAtExhibit variable does not matter here; we were looking for deeper engagement with contents.

We decided that a combined measure is important for data analysis:

0: the user did not touch the interface

1: the user touches the interface and quickly moves away

2: the user browses the contents but without further, deeper engagement

3-4: intermediate engagement

5: full engagement with contents, the user has accessed most aspects of the interface, reading into contents and engaging deeply with multiple contents within the system, e.g., reading texts, studying pictures, watching videos, interacting with the digital objects during the session.

\subsection{Data Processing and Analysis}

We analysed our data using both R statistics for quantitative analysis and NVivo for thematic analysis [23] across our datasets. We identified trends and patterns from visitor engagements, and explored the reasons behind the trends.

\section{Findings}

Here, we present the analysis and interpretation of our findings.

\subsection{Quantitative Data Analysis}

\subsubsection{Digital System Types and Quality}

Figure 3 shows the distribution of categories. The chart demonstrated that contemporary interactive 2D exhibits from the 1990s, the 'Multimedia' era, were the largest group of technologies at $47.2 \%$. Projection systems carrying narratives and interactivity were the second largest groups at $22.2 \%$. Multitouch $2 \mathrm{D}$, a technology emerging from 2010 stood at $11.1 \%$, equivalent to interactive 3D, also $11.1 \%$. There was very little adoption of Virtual Reality (5.6\%) and Augmented Reality (2.8\%). The chart demonstrated a small but emerging adoption of more advanced interactive systems, indicating that museums in China are transitioning into a new era.

The ranking of the quality of over 36 exhibits yielded a graph in Figure 3 (right). The quality follows that of a normal distribution. Although slightly skewed and tending towards high quality on average, most systems ranked in the medium quality. 

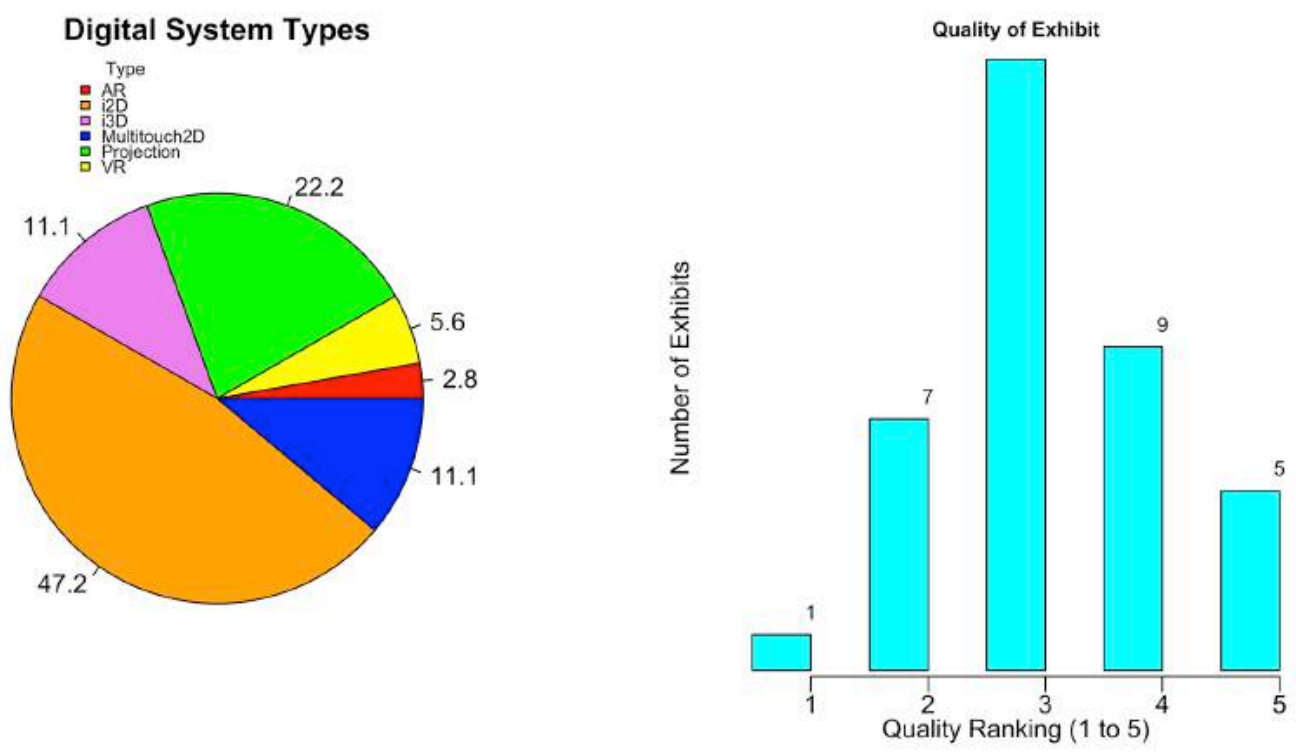

Figure 3. Digital systems and overall quality of exhibits.

We categorised the contents of these digital exhibits into three types:

1) Game: game-based exhibits such as jigsaw puzzles, linking games and Microsoft Kinect games, and etc.

2) Informational: introduction to the museums and its collections with text, images and videos as contents

3) Narratives: exhibits presenting narratives and storytelling

Figure 4 shows that $50 \%$ of the 36 exhibits we have observed were informational. Exhibits with narratives are the second largest group at 31\%. Only 19\% of exhibits provided are game-based. In terms of the quality, most 'Information' systems ranked medium and lower. Exhibits with games and narratives ranked medium but are ranked as having high qualities.
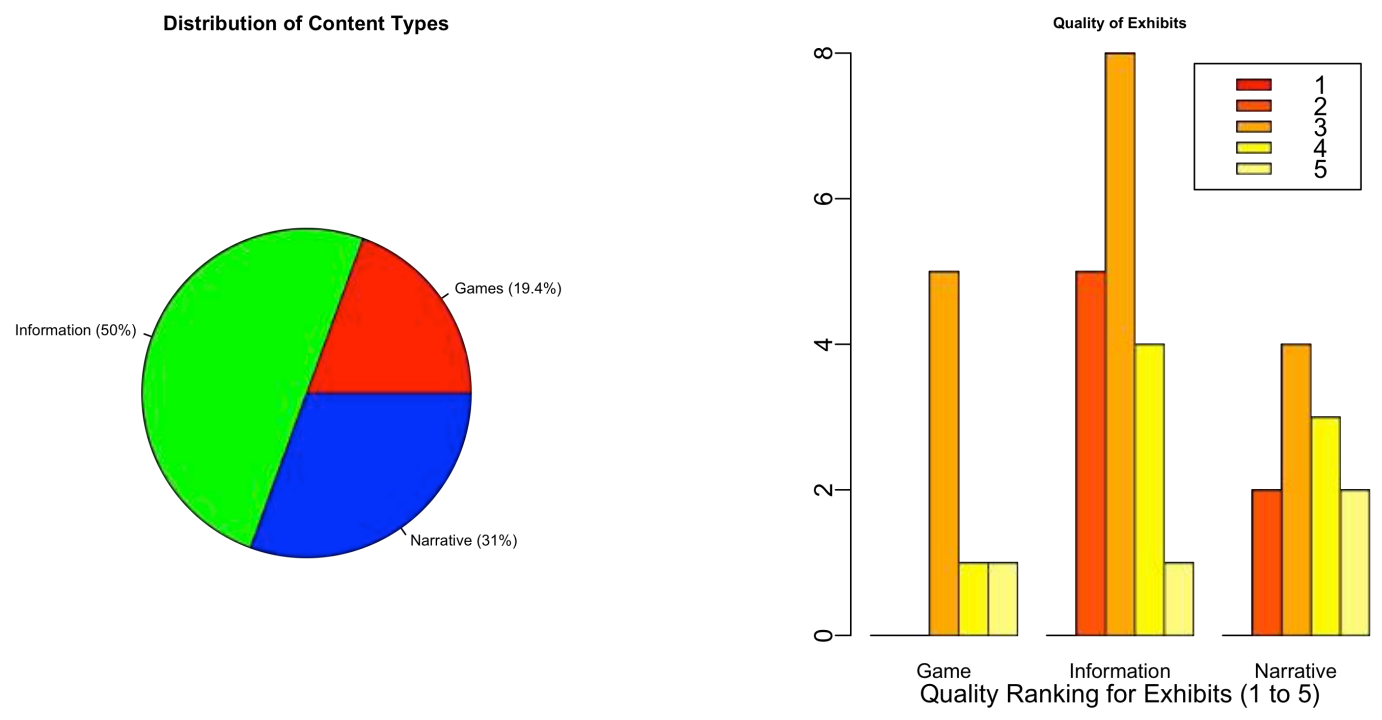
Figure 4. Distribution of contents of digital exhibits and their overall quality ranking.

\subsubsection{Demographics and Engagement}

Figure 5 illustrates our count of participant group sizes. 253 samples are single users, followed by 341 pairs who were not necessarily family units. The next two larger samples were 3 in a group (145 samples) and four in a group ( 41 samples), the graph ended with a long tail of larger group sizes.

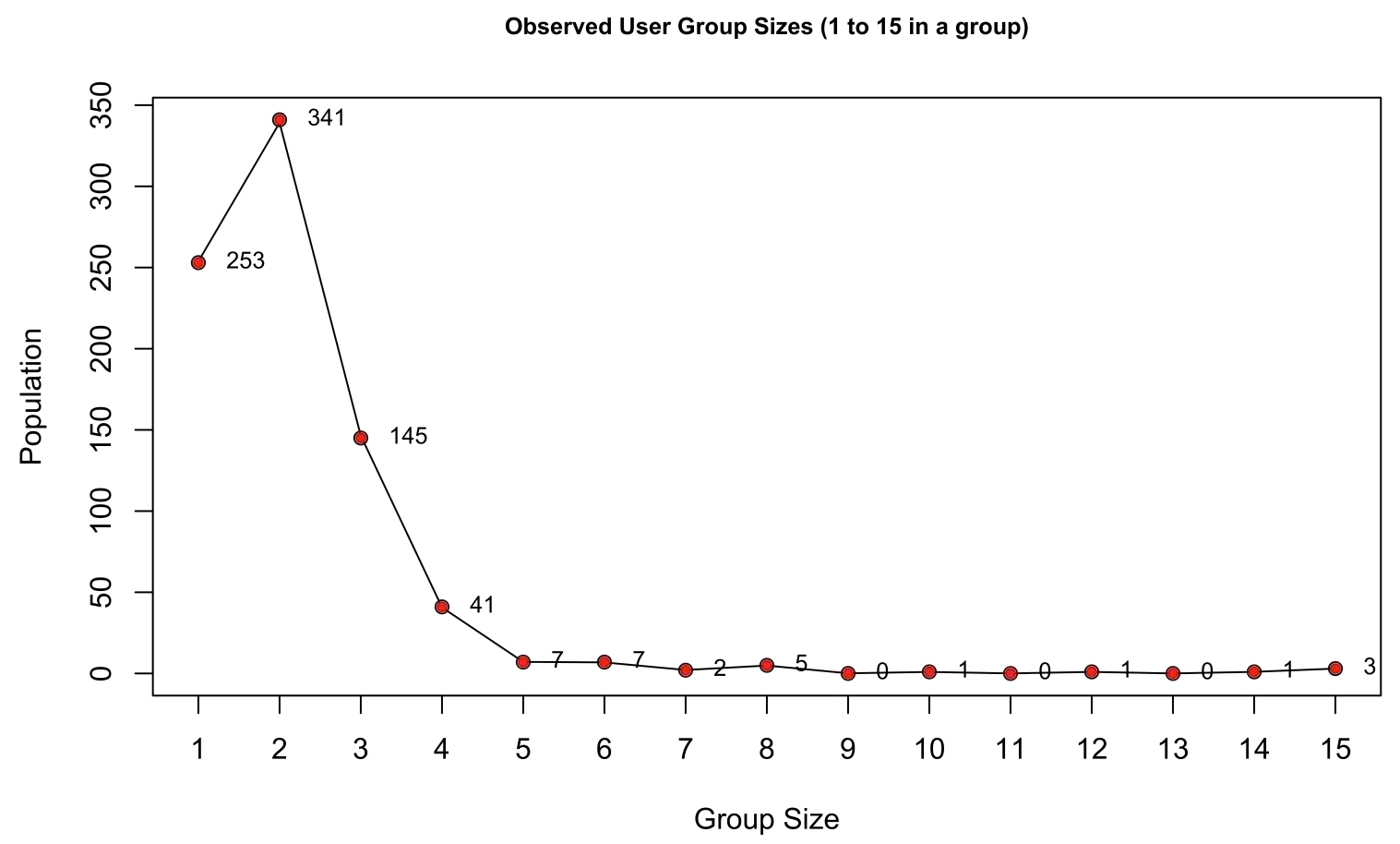

Figure 5. Observed participant group size plotted in descending order. 

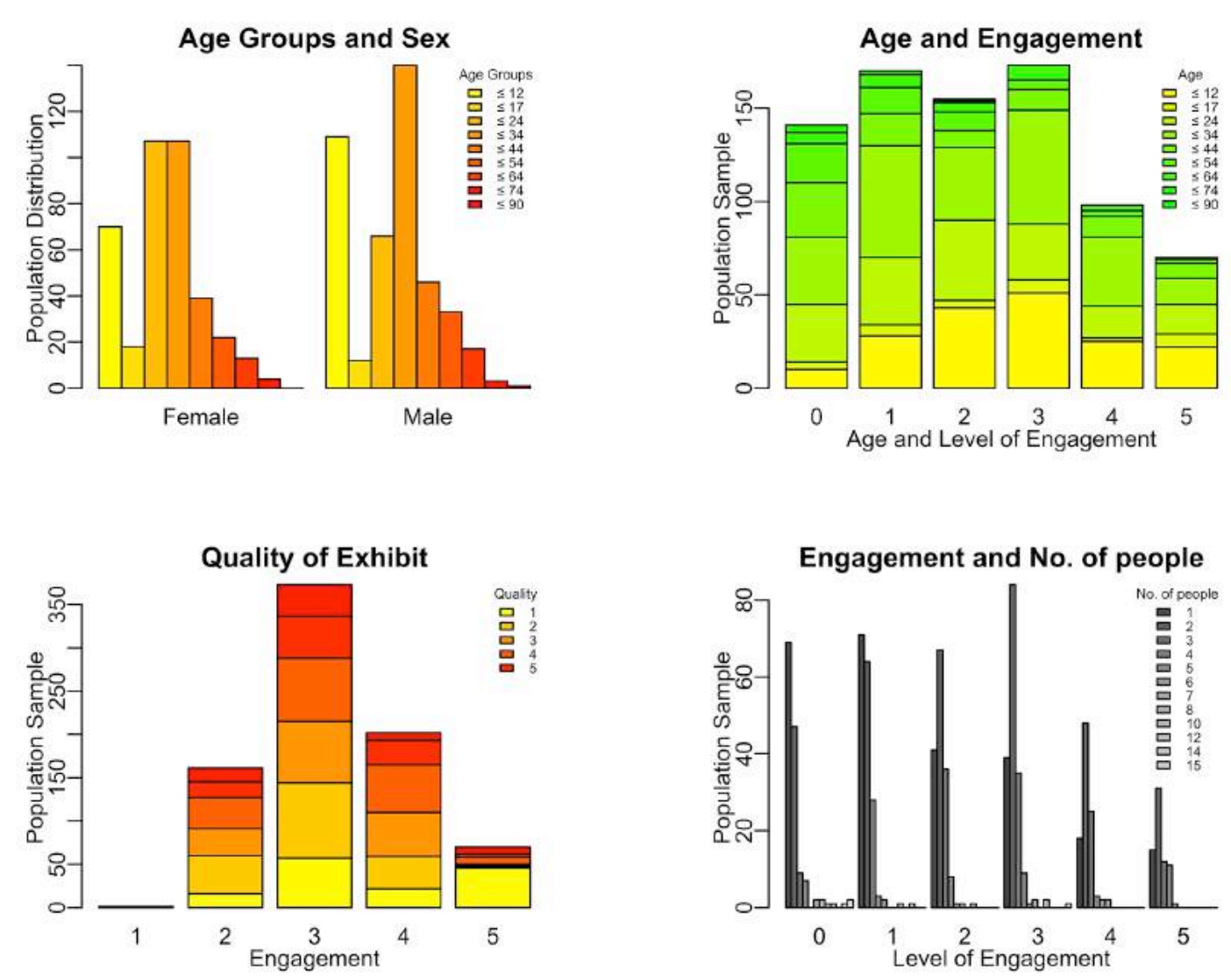

Figure 6. Sex and Age Groups (Top Left), Observed Age groups and Level of Engagement (Top Right), Observed Engagement and Quality of Exhibit (Bottom Left), Engagement and Group Size (Bottom Right).

In the Sex and Age group graph (Figure 6), there were 380 (47.09\%) female users and $427(52.91 \%)$ male users among which 209 are children and teenagers who were below the age of 17 (179 below 12 and 30 between 13 and 17). This is a significant number at $25.90 \%$ of the total sample. The bar to the far left shows a high proportion of children. The bar for children under 12 on the Male group shows us that there are a higher proportion of male children.

In the Age and Engagement chart (top right), the segregation of age and engagement seems to indicate that a larger proportion of users were not very engaged with the system (engagement $<3$ ). Younger people tended to be more engaged with digital contents although the level of engagement appears to average around the value of 3.

The box plot in Figure 7 indicated that younger people tended to be more engaged with digital systems than those who were above the age of 34 . However, the level of engagement seems to peak at an average of between 2 and 3 . This may indicate that the digital exhibits on display have not very engaging. 


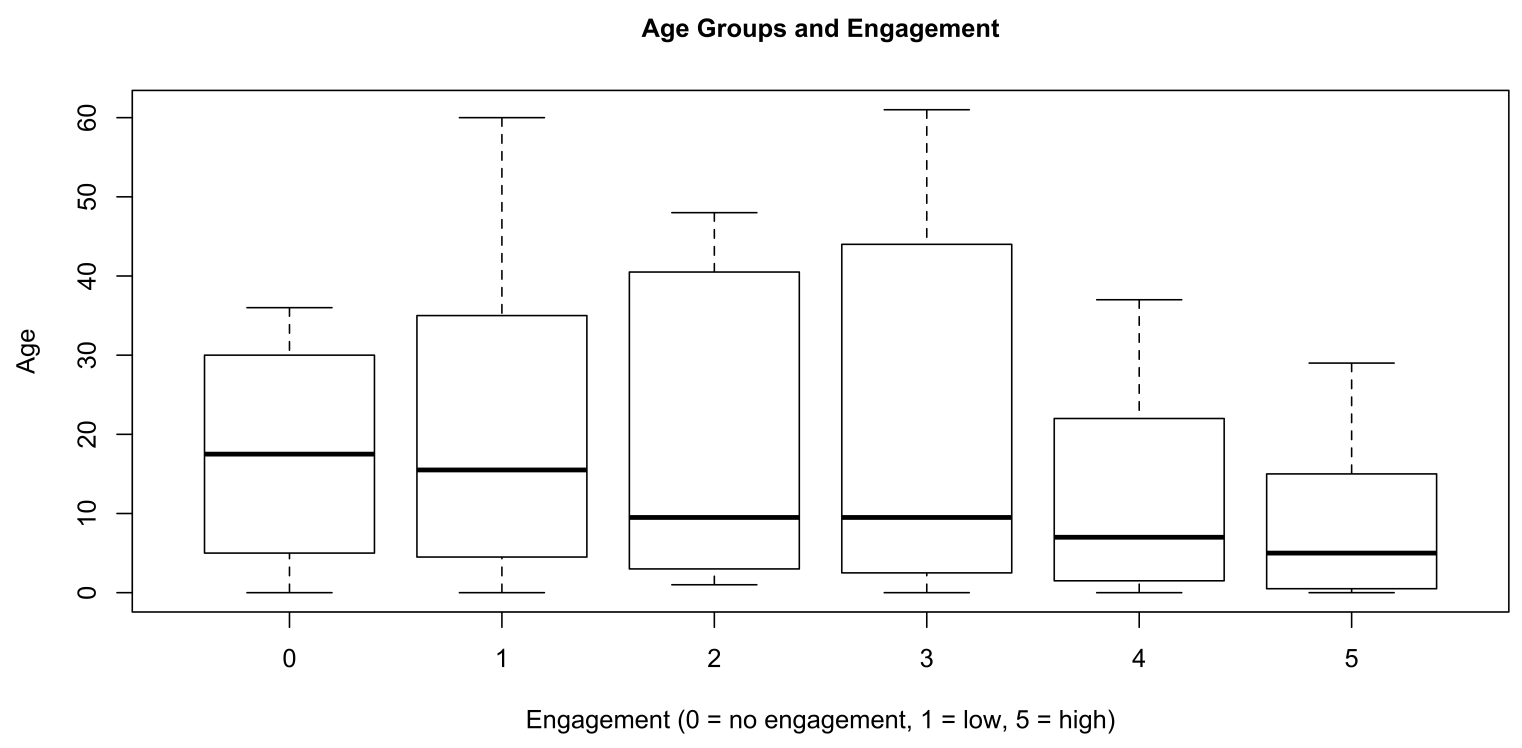

Figure 7. The box plot of age groups and the level of engagement.

\subsubsection{Visitor Access Time}

Figure 8 measures the length of access of the digital contents and the actual visitor time spent on that exhibit (top left), visitor access time and length of the exhibits (top right) and observed visitor engagement and actual time spent at the exhibit (bottom).

The graph on the top left compares the actual length of the exhibit if a user were to navigate through all the contents as compared to the actual time spent on exploring contents. There were outliers ( $>60 \mathrm{mins}$ ), but the average access time is at a mean of $\mu=2.67$, a value that falls far short of the systems and contents originally designed to engage with visitors.

We analysed visitor access time with a more detailed investigation of the length of exhibit. These were binned into the range of 8 bins from $0.5 \mathrm{sec}$ to $30 \mathrm{~min}$ (top right graph). The plot indicated that users tended to spend more time on exhibits with a length of 20 minutes or less, including the outliers. We also measured the level of engagement in comparison with the time that users have spent at the exhibits $0.5 \mathrm{sec}$ to $60 \mathrm{~min}$ ). The range of time between 2 to 20 minutes appears to be the most engaging overall, with 4 minutes being the highest overall. 

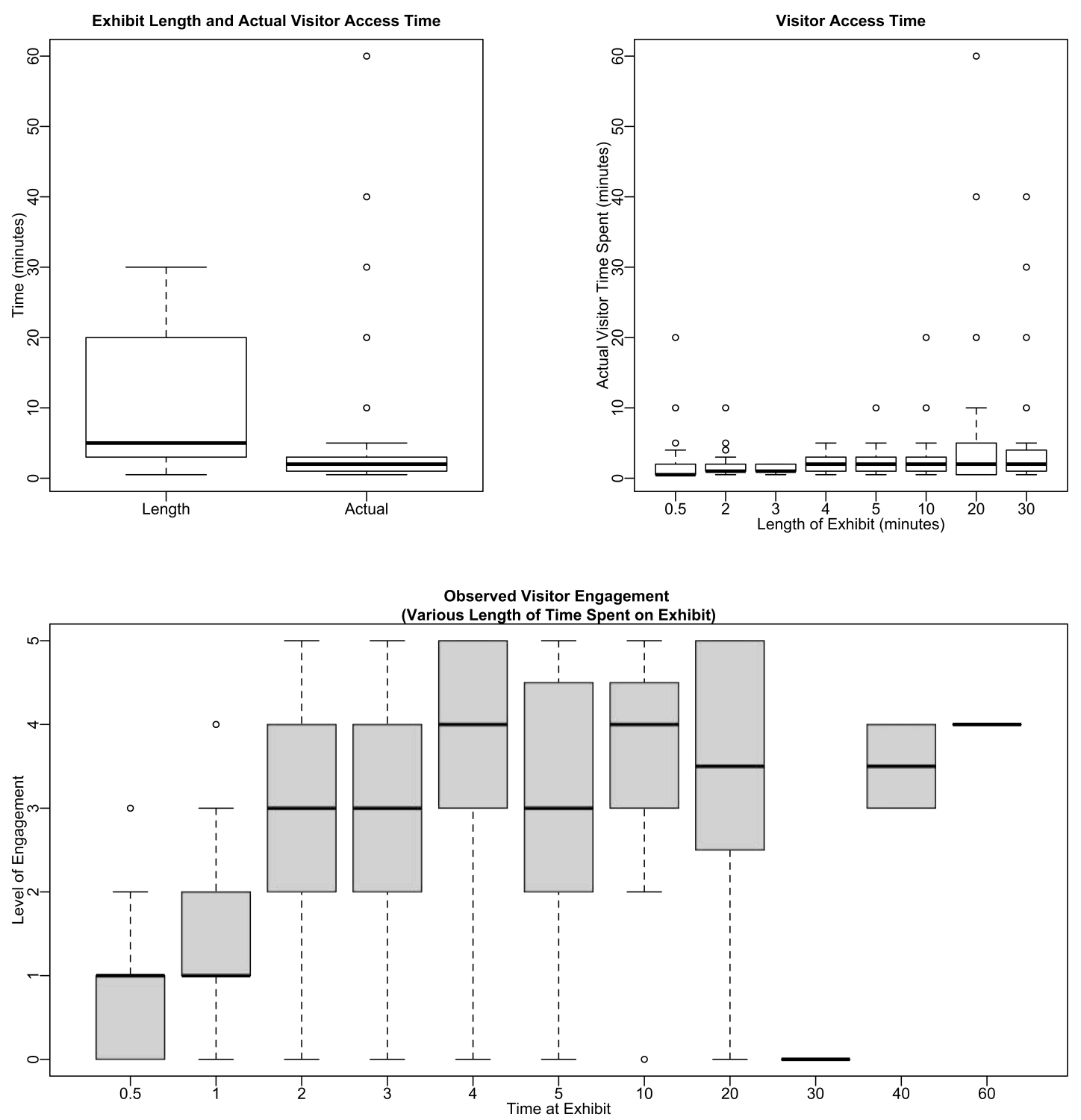

Figure 8. Length of time required to access all contents in the exhibits and actual visitor time spent on the exhibits (top left), visitor access time (top right), observed visitor engagement and time at the exhibit (bottom).

\subsubsection{Predictors for User Demographics, Groups and Access Time}

We conducted logistic regression to test the predictors of user demographics and the social aspects of using an exhibit using categorical variables ( $1=$ true, $0=$ false) user is an adult (Adult), family units (Family), discussions taking place (Discussions), sex, space is crowded (Iscrowded), use of exhibit attracts a queue (AttractsQueue), and how long the queuing user uses the system eventually (Queued). We used access time (retention) as predictors for determining the likelihood of our categorical variables. 

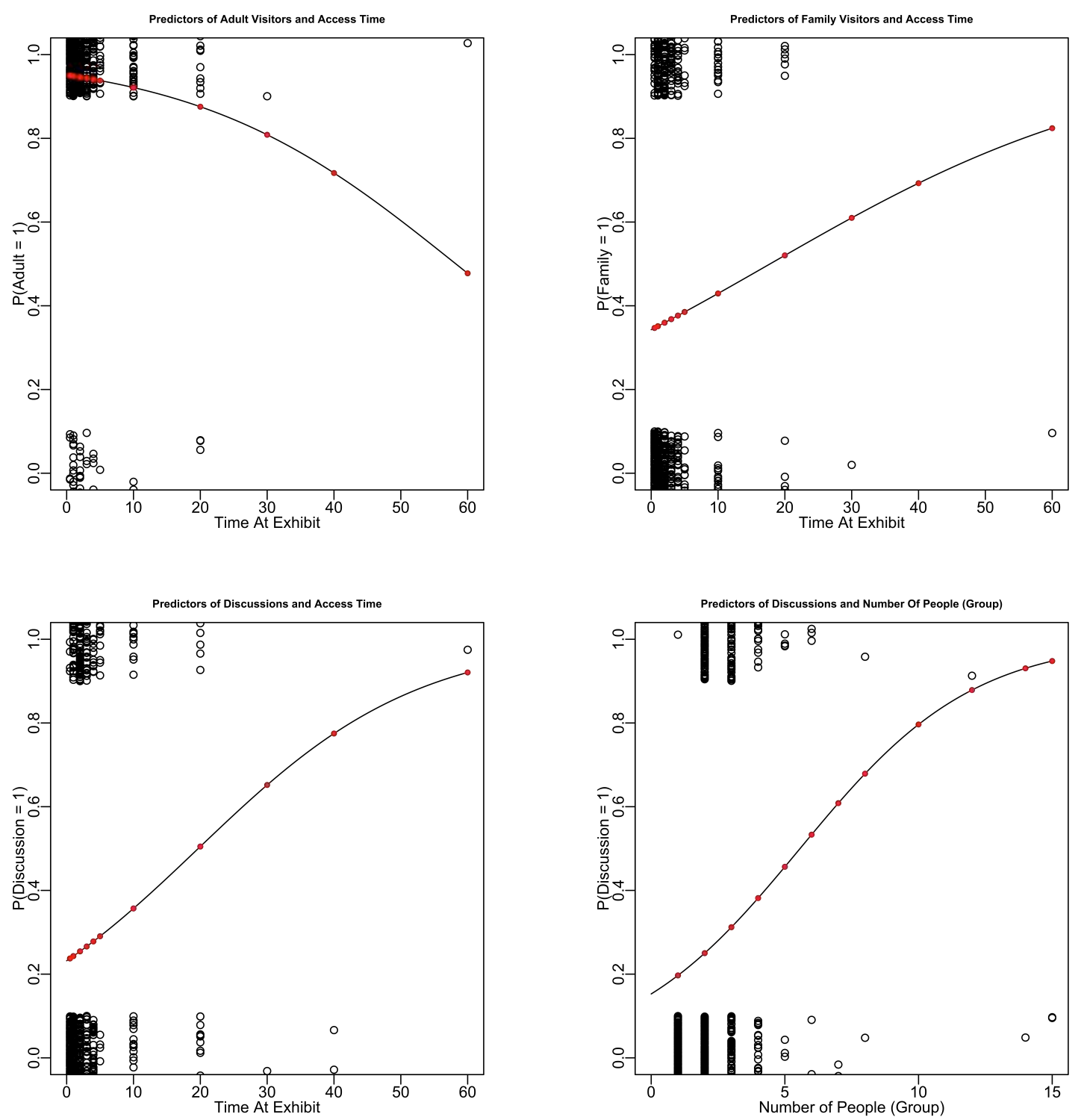

Figure 9. Predictors of access time and visitor demographics and groups.

Predictors of adult visitors and access time has a p-value of $\mathrm{p}<0.0001$ with the time spent using the exhibit being negative, indicating that adults on average tended to spend less time than children and teenagers (age $<18$ ). The summary coefficients indicated that for every 1 unit change in TimeAtExhibit, there is a log odds of the user not being an adult by -0.05 . Converting this into a scale in view of access time, for a unit increase in the variable TimeAtExhibit means that the odds of not being an adult is increased by a factor of 0.95 .

Predictors of family visitors and access time is significant with $\mathrm{p}<0.05$ with the time spent at the exhibit being positive indicating that family visitors tended to spend more time at the exhibit. The summary coefficients indicated that for every 1 unit change in TimeAtExhibit, there is a log odds of the likelihood that the visitors are a family by 0.04 . A unit increase in the variable TimeAtExhibit means that the odds of the visitors being a family is increased by a factor of 1.04 . This summarises that family units tended to spend more time at the exhibit. 
Predictors of discussions occurring between visitors and the length of access time has a $p$-value of $\mathrm{p}<0.001$ indicating that discussions tended to lead to more access time at the system. The summary coefficients indicated that for every 1 unit of change in TimeAtExhibit, there is a log odds of the likelihood that discussions have taken place by 0.02, a unit increase in the variable TimeAtExhibit contributed to the odds of discussions taking place being increased by a factor of 1.10 .

Predictors of discussions occurring between members of the same group and the number of people in that group is significant at $\mathrm{p}<0.0001$ indicating that the number of people in a group tended to lead to more discussions when using the system. The summary coefficients indicated that for every 1 unit of change in NumberOfPeople, there is a log odds of the likelihood that discussions will take place by 0.31 . A unit increase in the variable NumberOfPeople means that the odds of discussions taking place will be increased by a factor of 1.36 .

We also discovered that male and female users tended to have equal time accessing digital exhibits. Crowded spaces do not influence nor affect the use of digital exhibits. The quality of a system does not prolong its use either. This is probably an issue with systems not having appropriate retention power. Predictors on a person taking the effort and time to queue up and the queuing person's subsequent use of the system is not significant, indicating that whilst a system may be attractive, its ability to retain users is weak.

\subsection{Factors Affecting Digital Exhibits and Sample Size}

We identified four factors which can negatively affect the use of digital exhibits, one of which relates to the popularity of the museum. Despite the quality of an interactive 3D system, the Lacquer Ware gallery attracted only 2 users in two hours, even on a weekend. The location of the digital exhibit also affects its use, e.g., an interactive 2D exhibit which allows visitors to create calligraphy works attracted only 3 visitors in two hours of observation as it was located at a dark, obscured corner at the immediate right side of the entrance, and the screen was embedded in an artificial stone tablet. Technological limitations and user expectations may also affect exhibit use. For example, a jigsaw puzzle exhibit which easily attracted a crowd, which collectively attempted to complete the game. The crowd thought that the system supports multitouch. Over 30\% of users left after realising that the system does not support multiuser interaction. Other technical flaws included the sensitivity of, or non-responsive touch screens, blank homepages, and latency issues. The fourth factor was ergonomics. Some systems had intriguing contents meant for children, but the height of the exhibits was beyond the reach of children, nor were they designed for those with certain disabilities.

\subsection{Qualitative Explanation of Observed Phenomena}

Our analysis indicated that family groups with children tended to spend more time at digital exhibits. We conducted a qualitative analysis to acquire a deeper understanding of the given phenomenon, and categorised their behaviours as follows:

1) Positive Communication: user discusses amongst themselves, and/or teaches them how to use, and/or interacted with the system together with them, the behaviour is regarded as having a positive communication

2) Negative Communication: if the user is interrupted by their parents/children/friends/partners, and/or did not get 
supported/encouragement when necessary, and/or was forced to leave, the behaviour is regarded as having a negative communication.

3) No Communication: if the user did not interact with their parents/children/friends/partners, and/or is left alone, this is regarded as having no communication.

The frequency of occurrence of 'Positive Communication' among family visitors outnumbered those with two or more adult visitors (Table 6 and 7), although the percentage of 'Positive Communication' among family visitors is slightly lower than that of adult visitors. We observed that 'Positive Communication' between adult visitors were discussions and collaborations whilst family visitors had more teaching and learning activities. These factors did prolong the visits.

\begin{tabular}{|c|c|c|c|c|}
\hline Theme & $\begin{array}{l}\text { Positive } \\
\text { Communication }\end{array}$ & $\begin{array}{l}\text { Negative } \\
\text { Communication }\end{array}$ & $\begin{array}{l}\text { No } \\
\text { Communication }\end{array}$ & TOTAL \\
\hline Frequencies & 131 & 50 & 88 & 269 \\
\hline Percentage & $48.7 \%$ & $18.6 \%$ & $32.7 \%$ & - \\
\hline
\end{tabular}

Table 6. Frequency of the occurrence of 'Positive Communication' and 'Negative Communication' among family visitors

\begin{tabular}{|c|c|c|c|c|}
\hline Theme & $\begin{array}{l}\text { Positive } \\
\text { Communication }\end{array}$ & $\begin{array}{l}\text { Negative } \\
\text { Communication }\end{array}$ & $\begin{array}{l}\text { No } \\
\text { Communication }\end{array}$ & TOTAL \\
\hline Frequencies & 119 & 6 & 101 & 226 \\
\hline Percentage & $52.6 \%$ & $2.7 \%$ & $44.7 \%$ & - \\
\hline
\end{tabular}

Table 7. Frequency of the occurrence of 'Positive Communication' and 'Negative Communication' among adult visitors

'Positive Communication' occurs when children discovered an exhibit and invited the parents to explore with them. They also asked questions about the exhibit's use and/or the contents of the exhibit. We also noted that teenagers were teaching their grandparents. This suggests that the younger generation tended to be more curious. They are more attracted to and motivated by digital exhibits. This explained why younger visitors were more engaged with digital exhibits as compared to adult visitors, even though their level of engagement were rated between 2 and 3.

We observed that mutual communication was infrequent between adult visitors. There were only 6 cases within the 'Negative Communication' category in which adult visitors visibly forced their friends or partners to leave. Amongst the 6, questions were ignored when friends or partners initiated a conversation or asked for help. There were 50 cases of 'Negative Communication', in which parents forced their children to leave, or gave negative responses when guidance was needed. The common reason as observed was that children were rushed from exhibits so that they could move on to the physical artefacts. In China at least, parents may regard physical artefacts and texts as more effective ways of learning than digital exhibits. We observed an example where a father commented to his son intending to prolong his stay to watch a projection that 'you will not remember the contents even though you have watched it'. This simple case may 
suggest that parents were doubtful of the pedagogical effectiveness of digital exhibits, thinking of digital technologies as a distraction rather than as a means for teaching and learning.

\section{Discussions}

Digital technology is a key factor in the creation of value for both society and the creative economy on many levels. The use of digital technology however, is not separate from the culture of use. Digital technology depended on culture and its use affects and shapes culture to a greater extent. The adoption and use of digital technologies therefore must be studied. In this article, we report on our investigation of the state of well-being of the adoption and use of cultural technologies within museums, an important sector within the creative cultural industry where knowledge is stored and contextualised at the juncture between China's two Five Year Plans. We believe that a broad evaluation exercise covering important sites scattered across large geographical regions will yield samples worthy of this cause, for a broad evaluation can provide a superior overview in contrast to a more focused evaluation often conducted at the device level or within a single institution.

In our evaluation, we first categorised the types of cultural technologies we have observed. In the study, we saw a transition towards newer types of interactive exhibits which are experienced-based, e.g., VR. We also noted storytelling and games in our data. Most exhibits however, are still largely in the informational category. Quality ranking leaned towards the medium range with very few high-quality systems in use. It is difficult to gauge how far China's museums have developed as we did not have snapshots of historical systems from the past. This study therefore, will provide a historical snapshot for future studies. In terms of demographics, we have seen more couples, i.e., 2 persons accessing digital exhibits, followed by single visitors and three in a group, and an equal distribution of groups across the sample data. There was a balanced visitor population between the sexes. Age groups tended to include a large number of children, and those who were between 18 to 33 years of age. There were, not surprisingly, a lower number of teenagers even when our observations were mostly at the weekends.

In terms of age and engagement with digital exhibits, children tended to engage more with digital exhibits, together with those in the 25 to 33 age groups. This was followed by those in the age range of between 18 to 24 . The level of engagement tended towards an average of 2, when 5 is the highest level of engagement. Younger people tended to be more engaged with digital contents. Those in the age group of between 2534 are the second most engaged overall, with those between 18 to 24 being moderately engaged.

The measure of engagement and the quality of exhibits appears to have an equal distribution. They are clustered around the level of engagement of around 3. There were very few low-quality systems, as contents and interfaces are largely standardised. We felt that there was little risk of bad contents or exhibits if unconventional systems are not adopted.

As expected, single users, and groups of 2 or 3 persons were distributed across the 5 levels of engagement. They seem to lean towards lower averages. Visitors in groups of threes ranked average in a normal distribution. Single users and groups of twos were more irregular, skewed towards lower level of engagements. We also observed that 
family visitors with children tended to spend more time at exhibits than adult visitors. Our observation of positive communication within groups related to discussions and collaborations were higher in the adult groups. However, family visitors, i.e., those with children had teaching and learning on top of discussions and collaborative exploration. Parent was teaching their children how to use the user interfaces and access contents. Younger children were also observed to guide their parents or grandparents in the use of digital exhibits. This explained the lengthier time observed in household groups. We have also observed that most adult visitors do not communicate. Positive communication across all age groups is usually initiated by the younger visitors (18 and under), with teenagers teaching their grandparents or parents the use of the digital exhibits. This explained why younger generations tended to be more engaged with the exhibits. More negative observations were that parents were seen to steer their children away from digital exhibits. This perhaps can be attributed to a stereotypical view of digital technology as a distraction. Children were frequently rushed through digital exhibits.

In terms of visitor access time, we have observed that users tended to spend more time on exhibits with a length of 20 minutes. This included the outliers. We also measured the level of engagement in comparison with the actual length of time that users spent at the exhibit $(0.5 \mathrm{sec}$ to $60 \mathrm{~min})$. The range of time between 2 to 20 minutes appeared to be the most engaging overall, with 4 minutes being the highest overall.

We applied logistic regression to look at predictors for time spent on exhibits and found that adults tended to spend less time on digital systems while teenagers and children were spending more time. Family groups are the second strongest predictor of time spent on exhibits as discussed above. Discussions contributed to more time spent on exhibits. The number of people within groups is the strongest predictor for discussions taking place. Differences in the sexes, and whether an exhibit is crowded and does attract queues were not predictors of time spent on exhibits.

We also discovered negative factors affecting the use of digital exhibits. Whilst the quality and content could attract visitors, the popularity of the museum, visibility of the exhibits and technical flaws all contributed to a low frequency of access.

In conclusion, our analysis suggests that China's museums overall, are in an early transitional period in terms of the adoption of, and the development of cultural technology appropriate for visitor access. China's museums were quick to adopt and develop new technologies in the interactive 3D, VR and AR categories. Whilst this is a positive development in view of the initiation of the $13^{\text {th }}$ Five Year Plan, which will lead towards innovation, there certainly are opportunities for improvement. There is a general observation that systems are not designed with visitors in mind and that museums are largely object-centred rather than visitor-centred. This is an indication that digital technologies have not been properly adopted and, as a consequence its use was limited as far as cultural technologies are concerned. The transformative nature of digital technologies may not have been fully realised in China, but China's quick adoption and acceptance of digital technology will be a factor in the highly successful development of cultural technologies in the future.

\section{Acknowledgement}

The authors wish to express gratitude to the AHRC Centre for Digital Copyright and IP Research in China and the Ningbo Science and Technology Bureau for supporting the 
project (Grant Reference: AH/N504300/1).

\section{References}

[1] Ministry of Culture of the People's Republic of China, Cultural Technology Innovation Plan during 13th Five Year period from Ministry of Culture (文化部“十三五 ”时期文化科技创新规划), 2017.

[2] The State Council of the People's Republic of China, 13th Five Year Planning for Cultural Development and Reform, 2017.

[3] R. Parry, Digital Heritage and the Rise of Theory in Museum Computing, in: R. Parry (Ed.), Museums a Digit. Age, Routledge, Oxon, 2010: pp. 603-625.

[4] G. MacDonald, Change and challenge: museums in the information society, in: L. Karp, C.M. Kreamer, S.D. Lavine (Eds.), Museums Communities Polit. Public Cult., Smithsonian Press, Washington, 1992: pp. 158-181.

[5] E. Hooper-Greenhill, Museums and communication: an introductory essay, in: E. Hooper-Greenhill (Ed.), Museum, Media, Messag., Routledge, London, 1995: pp. 112.

[6] H.S. Hein, The Museum in Transition: A Philosophical Perspective, Smithsonian, 2014. https://books.google.co.uk/books?id=tqRqBgAAQBAJ.

[7] Lynn D. Dierking, J.H. Falk, Audience and accessibility, in: S. Thomas, A. Mintz (Eds.), Virtual Real Media Museum, American Association of Museums, Washington, 1998: pp. 57-70.

[8] D. Vom Lehn, C. Heath, Accounting for New Technology in Museum Exhibitions, Int. J. Arts Manag. 7 (2005) 11-21. http://www.jstor.org/stable/41064849.

[9] M. Economou, The Evaluation of Museum Multimedia Applications: lessons from research, in: R. Parry (Ed.), Museums a Digit. Age, Routledge, Oxon, 2010: pp. 523542.

[10] E. Ch'ng, The Mirror Between Two Worlds: 3D Surface Computing Interaction for Digital Objects and Environments, in: Digit. Media Technol. Virtual Artist. Spaces, IGI Global, Hershey, PA, 2013.

[11] E. Ch'ng, N. Cooke, User study on 3D multitouch interaction (3DMi) and gaze on surface computing, 2015. doi:10.1007/978-3-319-20898-5_41.

[12] S.A. Yoon, K. Elinich, J. Wang, C. Steinmeier, J.G. Van Schooneveld, Learning Impacts of a Digital Augmentation in a Science Museum, Visit. Stud. 15 (2012) 157170. doi:10.1080/10645578.2012.715007.

[13] R.E. Grinter, P.M. Aoki, M.H. Szymanski, J.D. Thornton, A. Woodruff, A. Hurst, Revisiting the visit:, in: Proc. 2002 ACM Conf. Comput. Support. Coop. Work CSCW '02, ACM Press, New York, New York, USA, 2002: p. 146. doi:10.1145/587078.587100.

[14] E. Ch'ng, Special Issue on Virtual Heritage: Cultural Agents, Environments, and Objects Guest Editor's Introduction, Presence Teleoperators Virtual Environ. 24 (2015) iii-vii. doi:10.1162/PRES_e_00228.

[15] A. Lewis, What can we learn from watching groups of visitors using digital museum exhibits?, Victoria and Albert Museum. (2014). http://www.vam.ac.uk/blog/digitalmedia/digital-exhibits-observational-research (accessed August 1, 2017).

[16] E. Hornecker, M. Stifter, Learning from interactive museum installations about interaction design for public settings, in: Proc. 20th Conf. Comput. Interact. Spec. Interes. Gr. Aust. Comput. Interact. Des. Act. Artefacts Environ. - OZCHI '06, ACM Press, New York, New York, USA, 2006: p. 135. doi:10.1145/1228175.1228201. 
[17] S. Dong, X. Wang, S. Xu, G. Wu, H. Yin, The development and evaluation of Chinese digital science and technology museum, J. Cult. Herit. 12 (2011) 111-115. doi:10.1016/j.culher.2010.10.003.

[18] T. Yin, Digital Museum in "New Museology" Context, Zhongguo Bowuguan (China Museum). 4 (2005) 36-40.

[19] G. Chen, Digital Museum Concept, Feature and Development Model Analysis (数字博 物馆概念、特征及其发展模式探析), Zhongguo Bowuguan (Chinese Museum). 3 (2007) 88-93.

[20] Y. Zheng, The Application and Planning Requirements of Multimedia Technologies in Museum Display (多媒体技术在博物馆中的展示), Cult. Artefact World (Wenwu Shijie). 4 (2008) 65-67.

[21] Y. Liu, The Reasonable Use of Digital Media Technologies in Museum Displays (数字 媒体技术在博物馆展示中的合理应用), Fudan University, 2012.

[22] H. Liu, X. Zhu, Y. Gao, Museum Digitisation Construction Analysis - Survey into the Public Perception and Use of Museum (博物馆数字化建设探析——公众对博物馆的认 知和使用状况调查), in: China Research Institute for Science Popularization (Ed.), Proc. 19th Natl. Conf. Theor. Study Sci. Pop. Int. Forum Commun., Popular Science Press, Beijing, 2012: pp. 343-349.

[23] G. Guest, K.M. MacQueen, E.E. Namey, Applied Thematic Analysis, SAGE Publications, Inc, 2012. 